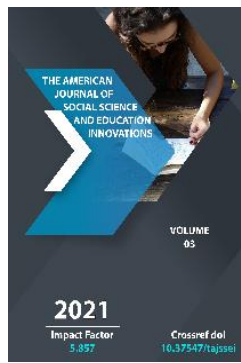

\title{
Combination Of Modern Science And Artificial Intelligence
}

Tashbabaey Maksud Djalilovich

Teacher of Denau Interpneurship and pedagogy institute, Uzbekistan

Journal Website:

https://theamericanjou

rnals.com/index.php/ta

jssei

Copyright: Original content from this work may be used under the terms of the creative commons attributes 4.0 licence.

\section{ABSTRACT}

This article deals with the scientific basis of the interrelationship between science and artificial intelligence at a time when scientific innovations are evolving rapidly. Besides there is also concept of research and future prospects in the field of artificial intelligence. How the revolutionary changes in science today will use the potential of artificial intelligence in the future shows the interdependence and prospects for the development of technology and human thinking.

\section{KEYWORDS}

Science, artificial intelligence, engineering, computer, computer, technology, research, cyber society.

\section{INTRODUCTION}

Humanity has passed a long historical path before reaching the current stage of development. Especially in recent years, scientific discoveries have been made in the field of science that was unique for centuries. The fact that the main parts of inventions are related to artificial intelligence has created a new stage in the development of modern science. To this day, individual research centers for artificial intelligence are operating in developed countries. For example: in the USA: Massachusetts Institute of Technology, Institute for Machine Intelligence Research, in Germany: German Research Center for Artificial Intelligence, in Japan: National Institute of Modern Industrial Science and Technology, in Russia: Scientific Council on Artificial Intelligence Methodology of the Russian Academy of Sciences and other major centers belong to them. 
According to the famous scientist V.A. Lektorsky, "The large-scale development of the Nano industry sphere transforms the human being as" a kind of biosocial being living simultaneously in different worlds: not only in the world of physical and biological processes, but also in the world of mineral resours, not only in the natural, but also in the artificial world ". only in the natural, but also in the artificial world. " [P. 1.6]. Indeed, by the 21st century, the development of technology has led to a change not only in the lives of people, but also in the whole of existence.

The second half of the twentieth century will lead to new revolutionary changes in science. These changes are often described in the literature as a scientific and technological revolution. These changes are due to the fact that after the Second World War, the achievements of science in economically developed countries will be applied in practice on an unprecedented scale - in industry, agriculture, healthcare, and everyday life. Science has made tremendous changes in energy (nuclear power plants), transport (automotive, aviation), electronics (television, telephony, computers). In the last decades of the last century, the development of science and technology entered the stage of the information (computer) revolution. The peculiarity of this stage is that information has become one of the most important resources for the development of society.

Thus, high technologies associated with science, and following with general education, now determine the level of civilizational development of any society. The distance between scientific discoveries and their implementation is shortened as much as possible. If earlier it took 50-100 years to find ways to practically use the achievements of science, now this can be done in 2-3 years, or even in a much shorter period [p. 2.206]. From the above data, it becomes clear that the future of humanity cannot be imagined without technology. The theory that electronic computers at a certain point in time will equal the capabilities of the human brain was first proposed by the English mathematician, logician, cryptographer Alan Turing. In his 1947 lecture, Intelligent Machines, Turing pondered whether a mechanism could find intelligent behavior. In 1950, his work Computing Machines and Perception was published, which demonstrated the method of "intelligent behavior" of the machine, and then the "Turing Test", or "game of imitation". The standard interpretation of this test is: "A person interacts with a computer and a person. Within five minutes, he should, based on answers to questions, determine who he is talking to: a person or a computer program." systems can freely pass his test, but this did not happen [3:67].

The capabilities of artificial intelligence systems differ in a very wide range. Currently, the capabilities of artificial intelligence are effectively used, including in the management of air, land and water transport, in the provision of expert services for individual medical services, in the provision of educational services, in industry and other areas that can be comparable to human intelligence.

In developed countries, artificial intelligence tools are used not only in the scientific direction, but also as the main driving force of the economy. The creation of artificial intelligence and the effective use of its capabilities should become the main direction of science. Because there are no clear rules for solving many problems, because of which the human mind does not have the ability to think fully and perfectly. This fully applies to all spheres of human mental activity, as well as to such an emotional sphere as logical thinking. We don't know how visual and auditory images move in our minds. While a person performs mental activity and makes appropriate decisions, he is not aware of the algorithm that 
occurs in the brain when such activity is performed [4. with. 114].

The main goal of creating artificial intelligence is to develop a model of rational human behavior at the level of consciousness. In turn, this allows for a deeper understanding and appreciation of artificial intelligence. At the same time, a knowledge base and powerful expert systems were created, covering thousands of rules and capable of solving certain problems better than any human mathematician.

Artificial intelligence is, first of all, the science and technology of creating intelligent machines, especially intelligent computer programs; secondly, it is the property of intelligent systems to perform creative tasks that have traditionally been considered human rights and privileges. Artificial intelligence serves the same function as using computers to understand human intelligence, but does not have to be limited to biologically accurate methods.

The problem of creating artificial intelligence and its widespread implementation has long worried people. In 1642, the French mathematician, physicist and philosopher Vlez Pascal invented the first calculating machine capable of performing calculations without human intervention. His calculating machine was improved in 1670 by the German mathematician and philosopher Gottfried Wilhelm Leibniz, who created an adding machine capable of performing all four arithmetic operations.

In 1812, Charles Babbage, dean of the mathematics department at the University of Cambridge, invented an "analytical engine" that not only performed arithmetic operations, but also remembered the initial, intermediate and final results and could repeat the calculation cycle if necessary. Then, in 1869 , the
English scientist William Stanley Jevons built a new logic machine based on formalized logic, developed in more detail than Leibniz's logical calculations, called the "logic piano." \{5. 117122b\}

At a conference held at the University of Dartsut in 1956, John McCartney said: "Artificial intelligence researchers have the right to use methods that can be observed in humans when they are needed to solve certain problems. Commenting on his definition, John McCarthy states:

"The problem is that we cannot yet determine exactly what computing processes we want to call intelligent. We can understand some of the mechanisms of intelligence and some not. Therefore, within the framework of this science, intelligence is understood as the computational component of the ability to achieve goals in the world. At the same time, there is a point of view that intelligence can only be a biological phenomenon. $\{6.147 \mathrm{C}\}$.

The history of artificial intelligence as a new scientific direction began in the middle of the twentieth century. By this time, many foundations had been laid for its emergence: philosophers have long been discussing the process of understanding human nature and the world, neurophysiologists and psychologists have developed a number of theories related to human consciousness and thinking, and economists and mathematicians have begun to study optimal calculations and formal representations of world knowledge. And finally, the mathematical theory of computation was laid - the foundations of the theory of algorithms and the first computers were created. The capabilities of new machines in terms of computing speed turned out to be higher than that of humans, so the question in the scientific community was closed. 
What is the limit of the capabilities of computers and can these machines reach the level of human development? In 1950, one of the pioneers of computing, the English scientist Alan Turing, described a process called the Turing test that allows us to determine the moment when a machine can be equated with a person in terms of intelligence, writes an article entitled "Can a machine think?"

Today, artificial intelligence does not have clear rules for solving many problems, so the human mind is limited in its ability to think completely and ideally. This fully applies to all areas of human mental activity, including the emotional sphere, for example, logical thinking. We do not know how the images of sight and hearing move in our minds. As long as a person performs mental activity and makes appropriate decisions, he does not understand the algorithm that arises in the brain when performing such an activity.

The main goal of creating artificial intelligence is to develop a model of rational human behavior at the level of consciousness. In turn, this allows for a deeper understanding and appreciation of artificial intelligence. The potential of artificial intelligence systems is quite wide. At the same time, a knowledge base and powerful expert systems were created, covering thousands of rules and capable of solving certain problems better than any human mathematician. There are computer systems that can read newspaper fonts with a human voice and perform quality translations of technical literature.

The philosophical aspect of artificial intelligence includes methodological guidelines and epistemological implications for the creation of machines that can perform cybernetic modeling of intellectual processes and data processing similar to the activities of the human mind.
In conclusion, we can say that the 21st century in the history of science will remain as the age of information and technology, as an integral part of human civilization. All means of artificial intelligence are aimed primarily at making life easier for people, more efficient organization of their work, work in all areas.

Today, when every country strives for innovation in science, it is necessary to conduct scientific research in the field of artificial intelligence. The processes associated with the coronavirus pandemic in the world in 2020 have shown that science enlightens humanity and, in mutual understanding, determines the future of its activities.

\section{REFERENCES}

1. Lectorskiy V.A. Possibilities of human science. Questionary Philosophy. 2015. № 3-16 p.

2. Shermukhamedova N.A. Philosophy T. Publisher. $2012 \mathrm{y}$.

3. Turing A. Can machines think ? - M.: physmathlit, 1960. $-67 \mathrm{p}$.

4. Prigojin I., Nicolis G. Пригожин И., Николис Г. Cognition of the complex. Introduction. Translation from English. Ed. 3, add. - M .: Publishing house LCI, 2008 .-- 114 p.

5. 5. Nikitin A. E. Artificial intelligence, in the light of the concepts of Heinz von Foerster Pomeranian University Bulletin: Copy

6. Martin J. The Telematic Society. Challenge of the near future // New technocratic wave in the West. - M., 1986.147 C. 